\title{
Turnover of lymphocytes and conceptual paradigms in HIV infection
}

\author{
Guido Silvestri and Mark B. Feinberg \\ Emory University School of Medicine and the Emory Vaccine Center, \\ Atlanta, Georgia, USA
}

\begin{abstract}
Prevailing views concerning the pathogenic mechanisms of AIDS have shifted from models that focus primarily on direct HIV-mediated killing of $\mathrm{CD}^{+} \mathrm{T}$ cells to models that emphasize the pathogenic role of generalized immune system activation. The observation that increases in $\mathrm{T}$ cell turnover seen in HIV-infected individuals primarily reflect increased proliferation of effector-memory $T$ cells (see the related article beginning on page 956) supports the concept that chronic immune activation plays a prominent, if not predominant, role in the pathogenesis of AIDS.
\end{abstract}

J. Clin. Invest. 112:821-824 (2003). doi:10.1172/JCI200319799.

\section{Accelerated T cell turnover as a result of HIV-mediated killing of CD4 ${ }^{+} \mathrm{T}$ cells}

Despite almost twenty years of study and the accumulation of significant experimental insights, the fundamental mechanisms underlying HIVinduced $\mathrm{CD}^{+} \mathrm{T}$ cell depletion remain incompletely understood. A series of influential studies published in the mid-1990s described the rapid decay of plasma viremia following initiation of highly active antiretroviral therapy (HAART) and proposed that HIV infection is associated with a high rate of virus turnover and a short lifespan of virus-infected cells (1-3). These observations led to the formulation of a model of AIDS pathogenesis whose central postulate was that HIV infection of susceptible $\mathrm{CD}^{+} \mathrm{T}$ cells leads to their destruction at such a high rate that compensatory $\mathrm{CD}^{+}$ $\mathrm{T}$ cell proliferation is inadequate to maintain sufficient $\mathrm{CD}^{+} \mathrm{T}$ cell numbers, resulting in progressive func-

Address correspondence to: Mark B.

Feinberg, Emory Vaccine Center,

954 Gatewood Road, Atlanta,

Georgia 30329, USA.

Phone: (404) 727-4374; Fax: (404) 727-8199;

E-mail: mbf@sph.emory.edu.

Conflict of interest: The authors have declared that no conflict of interest exists. Nonstandard abbreviations used: highly active antiretroviral therapy (HAART); activation-induced cell death (AICD). tional compromise of the immune system (1-3). In this model, the increased levels of $\mathrm{T}$ cell turnover observed in HIV-infected patients are interpreted mainly as a homeostatic mechanism aimed at compensating for the loss of $\mathrm{CD}^{+} \mathrm{T}$ cells induced directly by HIV. As such, this model proposed that direct, HIV-mediated killing of $\mathrm{CD}^{+} \mathrm{T}$ cells is the central event in AIDS pathogenesis. That the level of ongoing viral replication (commonly referred to as viral load) correlates with rates of $\mathrm{CD}^{+}{ }^{+} \mathrm{T}$ cell depletion and disease progression in HIV-infected individuals (4) was originally interpreted as supporting the primacy of direct virus-induced $\mathrm{CD}^{+}$ $\mathrm{T}$ cell destruction in driving the pathogenesis of HIV disease.

\section{Accelerated $\mathrm{T}$ cell turnover as a} result of chronic immune activation The view that accelerated $\mathrm{T}$ cell turnover is a result of HIV-mediated killing of $\mathrm{CD}^{+} \mathrm{T}$ cells has been recently challenged by a number of observations that collectively suggest that $\mathrm{CD}^{+} \mathrm{T}$ cell depletion in AIDS is related not only to the direct, virusmediated death of infected $\mathrm{CD}^{+}{ }^{+} \mathrm{T}$ cells, but also to apoptosis of large numbers of uninfected "bystander" $\mathrm{T}$ cells in the setting of the pronounced state of chronic immune activation that follows HIV infection (5-13).
Indeed, accumulating evidence indicates that most of the excessive T cell destruction associated with HIV infection involves uninfected cells that die as a result of activationinduced cell death (AICD) rather than direct HIV infection (5-12). Based on these observations, an alternative paradigm has emerged that posits that increased levels of $\mathrm{T}$ cell proliferation reflect the state of chronic generalized immune activation that follows HIV infection (10, 12-14), rather than a compensatory homeostatic response acting to maintain $\mathrm{CD}^{+} \mathrm{T}$ cell numbers. The fact that HIV infects and directly kills $\mathrm{CD}^{+} \mathrm{T}$ cells, but not $\mathrm{CD}^{+} \mathrm{T}$ cells, while the rates of both $\mathrm{CD}^{+}$and $\mathrm{CD}^{+} \mathrm{T}$ cell turnover are consistently elevated in HIV-infected individuals supports the idea that immune activation is the major force driving elevated levels of $\mathrm{T}$ cell turnover during HIV infection. Consistent with this view, levels of $\mathrm{CD}^{+} \mathrm{T}$ cell activation have been shown to predict rates of disease progression as well as, or even better than, viral load itself does (11, $15,16)$. Further, the finding that viral load correlates with rates of $\mathrm{CD}^{+} \mathrm{T}$ cell depletion and progression to AIDS (4) could also be explained by a process wherein levels of HIV replication either determine or reflect the prevailing level of immune activation. An intriguing observation consistent with the idea that the HIVassociated $\mathrm{CD}^{+} \mathrm{T}$ cell depletion is not simply the direct result of the virus-mediated killing of $\mathrm{CD}^{+} \mathrm{T}$ cells alone is that simian immunodeficiency virus-infected (SIV-infected) sooty mangabey monkeys (a natural host for SIV infection), in which the infection induces only minimal levels of immune activation and bystander immunopathology, do not manifest $\mathrm{CD}^{+} \mathrm{T}$ cell depletion, despite chronic high levels of virus replication (17).

\section{Turnover of specific $T$ cell subpopulations in HIV infection}

In this issue of the JCI, Hellerstein and colleagues use a highly innovative technique for defining the dynamics of $\mathrm{T}$ 

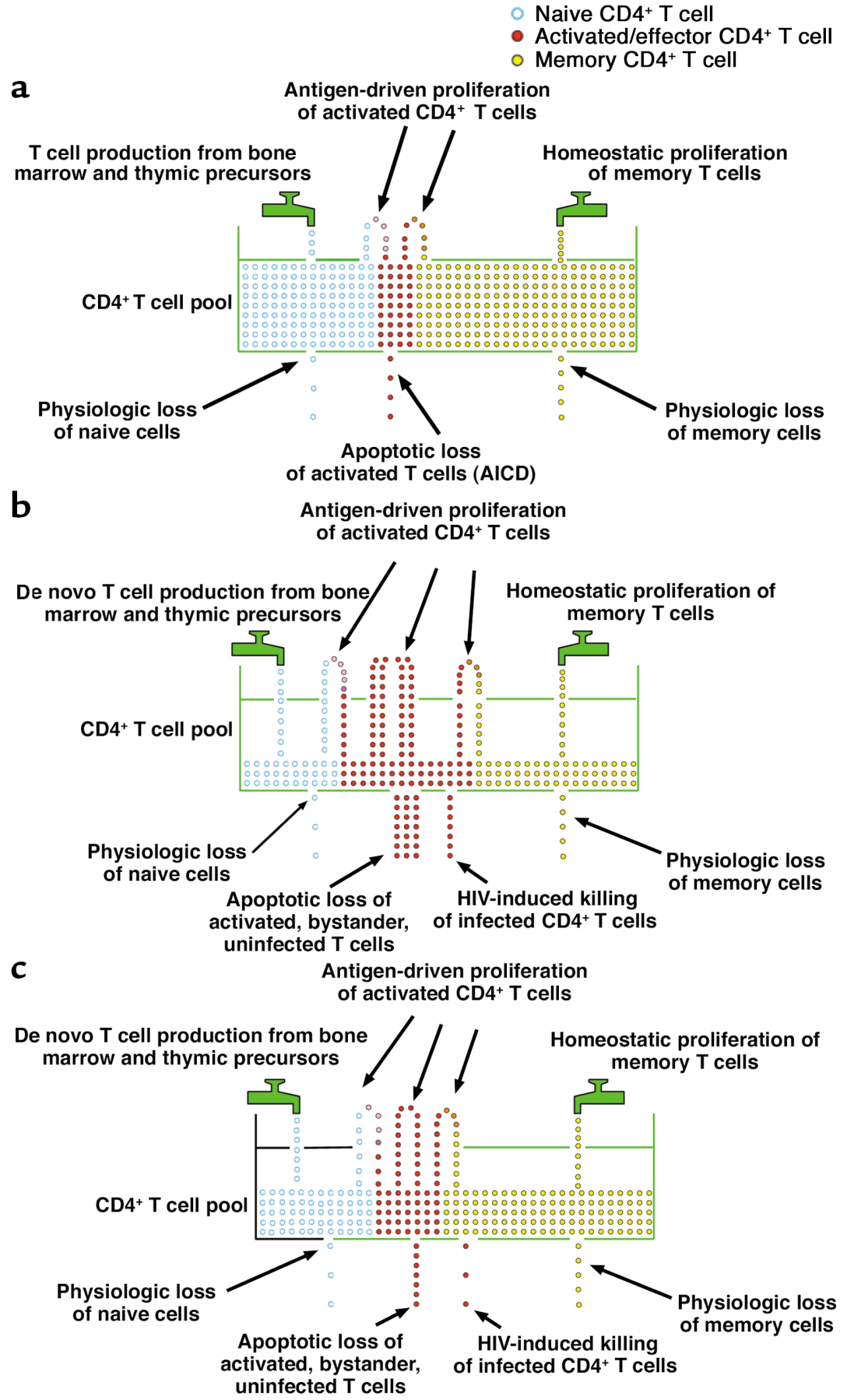

Figure 1

Turnover of specific CD4 ${ }^{+} T$ cell subpopulations (naive versus central memory versus effector memory) during HIV infection. (a) Uninfected controls. (b) HIV-infected individuals. (c) HIV-infected HAART-treated individuals. We present this model, which integrates of the work of Hellerstein et al. (18) with concepts emerging from references cited in this Commentary, as well as research not cited because of space limitations.

cell proliferation in vivo to delineate the relative turnover rates of different subpopulations of $\mathrm{CD}^{+}$and $\mathrm{CD} 8^{+} \mathrm{T}$ cells during HIV infection (18). The authors use administration of deuterium - a safe, stable, nonradioactive isotope of to unlabeled nucleosides was measured using gas chromatography and mass spectroscopy of hydrolized DNA specimens prepared from subpopulations of cells of defined immunophenotype, purified by multiparametric FACS analysis. The data thus generated are used to infer the kinetics of the cell population of interest, in this case, populations of naive and memory $\mathrm{CD}^{+}$and $\mathrm{CD}^{+} \mathrm{T}$ cells (19). The main finding of this report is that most of the increased $\mathrm{T}$ cell turnover (i.e., increased proliferation and accelerated cell death) observed in HIV infection involves a subset of memory $\mathrm{T}$ cells, of both $\mathrm{CD}^{+}$ and $\mathrm{CD}^{+}$lineage, that exhibit the turnover properties expected of socalled effector-memory cells, while normal levels of turnover are observed for both the naive and the so-called centralmemory $T$ cells (Figure 1). In addition, the authors observed that HAART is able to reduce both the level of $\mathrm{T}$ cell proliferation and the magnitude of $\mathrm{T}$ cell death in HIV-infected, successfully treated individuals. In all, the results reported by Hellerstein et al. strongly support the model that the increased levels of proliferation of effector-memory $\mathrm{CD}^{+}$and $\mathrm{CD}^{+} \mathrm{T}$ cells are related to the degree of prevailing immune activation and are not the result of a homeostatic mechanism striving to compensate for the loss of $\mathrm{CD} 4^{+} \mathrm{T}$ cells directly killed as a result of HIV infection. Whether and to what extent the fast-proliferating, fast-dying activated effector $\mathrm{CD}^{+} \mathrm{T}$ cells are directly killed by HIV awaits experimental elucidation, since the data provided by Hellerstein et al. are also compatible with the hypothesis that these cells are destined to die of AICD regardless of whether they are infected with HIV. This latter possibility is supported by the observation of a similarly expanded pool of fast-proliferating, short-lived $\mathrm{CD}^{+} \mathrm{T}$ cells that are not infected with HIV and that thus most likely die of AICD (18).

\section{Pathogenic implications of increased turnover of effector- memory $T$ cells}

The expansion of a pool of fast-replicating but short-lived effector cells, of both $\mathrm{CD}^{+}$and $\mathrm{CD}^{+} \mathrm{T}$ cell lineages (Figure 1b), carries a number of important implications for our understand- 
ing of the pathogenic mechanisms of HIV disease. First, the expansion of effector-memory cells may exert a continuous drain of the naive $T$ cell pool, which could, in turn, result in reduced capacity of the host immune system to generate primary responses to newly encountered antigens. Second, the expansion of such short-lived effector cells following HIV infection does not effectively lead to the establishment of a normal pool of true memory cells, i.e., the slow-proliferating, long-lived cells that show strong proliferative responses to recall antigens and that are believed to mediate protection from various infections, including lymphocytic choriomeningitis virus in mice (20) and HIV in humans (21). Third, the expansion of effectors that are able to produce large amounts of proinflammatory and proapoptotic cytokines may play a salient role in promoting the exaggerated bystander immunopathology associated with HIV infection. Finally, the generation of large numbers of activated $\mathrm{CD}^{+} \mathrm{T}$ cells that are known to be preferentially infected by HIV provides a fertile substrate for viral replication (21), thus creating the conditions for a vicious cycle in which more viral replication induces more immune activation, which in turn allows more viral replication, and so on.

The role of HAART in breaking this deleterious cycle of chronic virus replication and attendant immune activation is multifaceted (Figure 1c). On the one hand, HAART reduces the numbers of $\mathrm{CD}^{+} \mathrm{T}$ cells that are infected and directly killed by the virus, while on the other hand, HAART facilitates the re-establishment of normal $\mathrm{T}$ cell dynamics by decreasing the level of antigenic load and thereby indirectly reducing the level of $T$ cell activation and AICD. That HAART more likely acts to increase $\mathrm{CD}^{+} \mathrm{T}$ cell levels through this indirect mechanism is supported by the observation that an increase in $\mathrm{CD}^{+} \mathrm{T}$ cell counts that follows initiation of HAART is more strictly correlated, in certain clinical situations, with its salutary effect on immune activation and apoptosis than with direct effects to decrease levels of HIV replication $(16,22)$. These particularly illuminating clinical situ- ations include HIV-infected individuals with persistent suppression of viremia but no significant $\mathrm{CD} 4^{+} \mathrm{T}$ cell increase after initiation of HAART (so-called immunological nonresponders) and who exhibit evidence of persistent immune activation (22); and individuals with drug-resistant HIV variants who manifest sustained $\mathrm{CD}^{+}$ $T$ cell increases despite only partial suppression of viremia in the presence of HAART, in association with decreased levels of immune activation while continuing on therapy (23).

\section{Unanswered questions and future directions}

While the present study (18), in conjunction with other recently published studies, provides important clues as to how HIV infection leads to $\mathrm{CD}^{+} \mathrm{T}$ cell depletion and AIDS, many questions remain to be answered. In particular, we still lack an explanation of why HIV appears to be uniquely powerful in inducing a chronic state of immune activation (as opposed to other chronic viral infections such as hepatitis $\mathrm{C}$ virus), and why the HIVinduced immune activation is so disruptive of the proper overall functioning of the immune system. In this regard, it is important to determine the extent to which the pathogenesis of AIDS reflects the qualitative dysfunction of $\mathrm{T}$ cells above and beyond the evident numerical abnormalities in levels of $\mathrm{T}$ cell proliferation and death, and the consequent imbalance of the major $\mathrm{T}$ cell subsets (i.e., in naive versus memory-central versus memory-effector cells; see Figure 1). Finally, it is unclear whether and to what extent the suppression of the $T$ lymphocyte regenerative capacity contributes to the $\mathrm{CD} 4^{+} \mathrm{T}$ cell depletion that follows HIV infection $(9,19)$. Indeed, the fact that only $\mathrm{CD}^{+} \mathrm{T}$ cells, not $\mathrm{CD}^{+} \mathrm{T}$ cells, are severely depleted in HIV-infected individuals needs to be better understood, since this phenomenon may, in fact, not be a consequence of the HIV-mediated preferential killing of $\mathrm{CD}^{+} \mathrm{T}$ cells per se, but rather reflect an intrinsically higher susceptibility of $\mathrm{CD}^{+} \mathrm{T}$ cells to the deleterious effects of chronic immune activation, or an intrinsically lower capacity of $\mathrm{CD}^{+} \mathrm{T}$ cells to expand in order to maintain their proper homeostatic levels. At least some of these questions can be resolved with future application of the innovative and informative labeling techniques pioneered by Hellerstein and colleagues (18). Even better definition of the determinants and consequences of accelerated $T$ cell turnover during HIV infection will likely emerge from future longitudinal studies in which these labeling strategies are used in concert with FACS approaches with even higher resolution in defining and purifying specific subsets of $\mathrm{T}$ cells (24). With continuing advances in our understanding of the pathogenic mechanisms of AIDS and in the techniques available to study them, new therapeutic strategies will hopefully emerge that beneficially limit deleterious host responses, and not just levels of active HIV replication alone.

1. Ho, D.D., et al. 1995. Rapid turnover of plasma virions and CD4 lymphocytes in HIV-1 infection. Nature. 373:123-126.

2. Wei, X., et al. 1995. Viral dynamics in human immunodeficiency virus type 1 infection. Nature. 373:117-122.

3. Perelson, A.S., Neumann, A.U., Markowitz, M., Leonard, J.M., and Ho, D.D. 1996. HIV-1 dynamics in vivo: virion clearance rate, infected cell lifespan, and viral generation time. Science. 271:1582-1586.

4. Mellors, J.W., et al. 1996. Prognosis in HIV-1 infection predicted by the quantity of virus in plasma. Science. 272:1167-1170.

5. Finkel, T.H., et al. 1995. Apoptosis occurs predominantly in bystander cells and not in productively infected cells of HIV- and SIV-infected lymph nodes. Nat. Med. 1:129-134.

6. Gougeon, M.L., et al. 1996. Programmed cell death in peripheral lymphocytes from HIV-infected persons: increased susceptibility to apoptosis of CD4 and CD8 T cells correlates with lymphocyte activation and with disease progression. J. Immunol. 156:3509-3520.

7. Roederer, M. 1998. Getting to the HAART of HIV dynamics. Nat. Med. 4:145-146.

8. Haase, A.T. 1999. Population biology of HIV-1 infection: viral and CD4+ T cell demographics and dynamics in lymphatic tissues. Annu. Rev. Immunol. 17:625-656.

9. McCune, J.M. 2001. The dynamics of CD4+ T-cell depletion in HIV disease. Nature. 410:974-979.

10. Grossman, Z., Meier-Schellersheim, M., Sousa, A.E., Victorino, R.M., and Paul, W.E. 2002. CD4+ T-cell depletion in HIV infection: are we closer to understanding the cause? Nat. Med. 8:319-323.

11. Sousa, A.E., Carneiro, J., Meier-Schellersheim, M. Grossman, Z., and Victorino, R.M. 2002. CD4 T cell depletion is linked directly to immune activation in the pathogenesis of HIV-1 and HIV-2 but only indirectly to the viral load. J. Immunol. 169:3400-3406

12. Douek, D.C., Picler, L.J., and Koup, R.A. 2003. T cell dynamics in HIV infection. Annu. Rev. Immunol. 21:265-304.

13. Cohen, O.J., Kinter, A., and Fauci, A.S. 1997. Host factors in the pathogenesis of HIV disease. Immunol. Rev. 159:31-48.

14. Hazenberg, M.D., Hamann, D., Schuitemaker, H., 
and Miedema, F. 2000. T cell depletion in HIV-1 infection: how CD4+ T cells go out of stock. Nat. Immunol. 1:285-289.

15. Giorgi, J.V., et al. 1999. Shorter survival in advanced human immunodeficiency virus type 1 infection is more closely associated with T lymphocyte activation than with plasma virus burden or virus chemokine coreceptor usage. J. Infect. Dis. 179:859-870.

16. Hunt, P.W., et al. 2003. T cell activation is associated with lower CD4+ $\mathrm{T}$ cell gains in human immunodeficiency virus-infected patients with sustained viral suppression during antiretroviral therapy. J. Infect. Dis. 187:1534-1543.
17. Silvestri, G., et al. 2003. Nonpathogenic SIV infec tion of sooty mangabeys is characterized by limited bystander immunopathology despite chronic high-level viremia. Immunity. 18:341-352.

18. Hellerstein, M.K., et al. 2003. Subpopulations of long-lived and short-lived T cells in advanced HIV-1 infection. J. Clin. Invest. 112:956-966. doi:10.1172/ JCI200317533.

19. Hellerstein, M., et al. 1999. Directly measured kinetics of circulating $\mathrm{T}$ lymphocytes in normal and HIV-1-infected humans. Nat. Med. 5:83-89.

20. Wherry, E.J., et al. 2003. Lineage relationship and protective immunity of memory CD8 T cell subsets. Nat. Immunol. 4:225-234.
21. Migueles, S.A., et al. 2002. HIV-specific CD8+ T cell proliferation is coupled to perforin expression and is maintained in nonprogressors. Nat. Immunol. 3:1061-1068.

22. Paiardini, M., et al. 2003. Early correction of cell cycle abnormalities predicts a good immunological response to HAART in HIV-infected patients. AIDS. In press.

23. Douek, D.C., et al. 2002. HIV preferentially infects HIV-specific CD4+ T cells. Nature. 417:95-98.

24. De Rosa, S.C., Brenchley, J.M., and Roederer, M 2003. Beyond six colors: a new era in flow cytometry. Nat. Med. 9:112-117.

\title{
HATs off to Hop: recruitment of a class I histone deacetylase incriminates a novel transcriptional pathway that opposes cardiac hypertrophy
}

\author{
Yasuo Hamamori ${ }^{1,2,3}$ and Michael D. Schneider ${ }^{1,2,3,4}$ \\ ${ }^{1}$ Center for Cardiovascular Development, \\ ${ }^{2}$ Department of Medicine, \\ ${ }^{3}$ Department of Molecular and Cellular Biology, and \\ ${ }^{4}$ Department of Molecular Physiology and Biophysics, Baylor College of Medicine, \\ Houston, Texas, USA
}

\begin{abstract}
Histone acetylation, regulated by two antagonistic enzymes - histone acetyltransferases (HATs) and histone deacetylases (HDACs) - results in transcriptional changes and also plays a critical role in cardiac development and disease. A new study (see the related article beginning on page 863) shows that overexpression of the atypical transcriptional corepressor homeodomain-only protein (Hop) causes cardiac hypertrophy via recruitment of a class I HDAC. In contrast to the body of work on transcriptional mechanisms that drive cardiac hypertrophy, including class II HDACs, this report elucidates a novel growth-suppressing transcriptional pathway in cardiac muscle that opposes hypertrophic growth.
\end{abstract}

\section{J. Clin. Invest. 112:824-826 (2003). doi:10.1172/JCI200319834.}

Cardiac mechanical dysfunction poses a therapeutic challenge that is not effectively addressed by existing therapy. Heart failure represents the final common pathway for diverse disorders intrinsic to or impinging on the myocardium, including myocardial

\footnotetext{
Address correspondence to: Michael D. Schneider, Center for Cardiovascular Development, Baylor College of Medicine, One Baylor Plaza, Room 506D, Houston, Texas 77030, USA.

Phone: (713) 798-6683; Fax: (713) 798-7437;

E-mail: michaels@bcm.tmc.edu.

Conflict of interest: The authors have

declared that no conflict of interest exists.

Nonstandard abbreviations used: histone acetyltransferase (HAT); histone deacetylase (HDAC); homeodomain-only protein (Hop); serum response factor (SRF).
}

infarction and hypertension-induced cardiac hypertrophy. Pathological hypertrophy comprises defects in myocyte performance, fibrosis, and myocyte loss, compounded by insufficient replacement (1). Finding the signals and effectors that execute this phenotype has been a unifying theme for two decades of research into the molecular underpinnings of heart failure, encapsulated as the transcriptional activation of an ultimately adverse gene program, whose elements encompass contractile proteins, ion transport proteins, growth factors, cytokines, and regulators of survival (1). Among the best-proven nodal control points for gene regulation in hypertrophic myocardium is histone acetylation (2).
Together with other histone modifications, the consequent change in chromatin structure is a prerequisite for access of transcription factors to their target DNA (3-5).

The status of histone acetylation, at a given promoter, is determined by the balanced action of histone acetyltransferases (HATs) and histone deacetylases (HDACs). The essential role of a HAT protein in cardiac muscle was first proven by deletion of the coactivator p300, which perturbed heart development and cell proliferation (6). But p300 is an especially complex platform for factor-factor interactions, and it remains ambiguous which of its effects are contingent on HAT activity, which on recruitment of p300/CBPassociated factor - also a HAT protein - and which on other properties (7).

Three classes of mammalian HDACs have been discovered. Class I HDACs are expressed ubiquitously and are composed largely of the catalytic domain. Class II HDACs are highly expressed in striated muscle and contain extensions at the $\mathrm{N}$ - or $\mathrm{C}$-terminus (2). A third class comprises homologues of yeast Sir2 (8). Notably, class II HDACs can act as signal-responsive repressors of cardiac hypertrophy, inhibiting gene expression that is dependent on myocyte enhancer factor-2 $(1,2)$. Consistent with this repressive role, mutant mice lacking the class II HDAC9 are hypersensitive to hypertrophic signals (2).

\section{The Hop-less heart}

Homeodomain-only protein (Hop) is a new, unusually short homeodomain protein that was discovered by screening of expressed sequence tag databases for cardiac transcripts encoding novel homeobox genes $(9,10)$. Hop is 